\title{
Die verschiedensprachigen Ausgaben der Pastoralinstruktion: ein Vergleich
}

von Friedbelm Geller

I.

Die Pastoralinstruktion "Communio et Progressio " ist aus Teilarbeiten, die in verschiedenen Sprachen erstellt wurden, zusammengewachsen. ${ }^{1}$ Gleichsam als Ausdruck dieser internationalen Zusammenarbeit, wurde das Dokument - als erstes in der kirchlichen Praxis - gleichzeitig in mehreren Sprachen veröffentlicht: „Mit Nachdruck hatten Bischöfe aus verschiedenen Ländern gefordert, daß die Pastoralinstruktion neben dem authentischen lateinischen Text gleichzeitig auch in den wichtigsten Sprachen katholischer Länder veröffentlicht werden sollte. “2

Von der Ubertragung des deutschen Textes ${ }^{3}$ berichtet Hans Wagner, der selbst zum Ubersetzerteam gehörte: „Der nun veröffentlichte Text ... fußt also nicht auf einem einheitlichen und im Zusammenhang konzipierten Entwurf ... Die meisten Passagen sind aus dem englischen, französischen, deutschen oder italienischen Urtext ins Lateinische übersetzt worden. Die vorliegende deutsche Úbersetzung . . . wurde in mehrwöchigen Arbeitssitzungen auf der Grundlage einer Rohübersetzung von Prälat Anton Kochs in Rom von folgendem Arbeitsteam angefertigt: P. Dr. KarlHeinz Hoffmann SJ, Rom; Prälat Anton Kochs, Bensberg; Prälat Karl-August Siegel, Hamburg; und Dr. Hans Wagner, München. "4

Úber das selbstgesteckte Ziel des Teams heißt es: „Das Bestreben der Ubersetzung war es, das authentische Dokument zu vermitteln, dabei so wörtlich und so nahe am lateinischen Text zu bleiben wie möglich und trotzdem eine gute deutsche Fassung zu erstellen .... Wo der deutsche Text scheinbar keine Obersetzung des lateinischen Wortlautes zu sein scheint, rührt das daher, daß als Ubersetzungsgrundlage in mehr als der Hälfte aller Absätze nicht nur der lateinische Text, sondern auch der dem Lateinischen zugrundeliegende Urtext zu Rate gezogen wurde; in zahlreichen Fällen ging man auf die gesamte Entstehung einer Passage zurück, um möglichst präzise Sinn und Inhalt einer Aussage zu erfassen. ${ }^{{ }_{4}}$ Anton Kochs, Autor der Rohübersetzung, definiert ein ähnliches Ziel: „Die deutsche autorisierte Öbersetzung bemüht sich, das lateinische Dokument in enger Anlehnung an die Fachsprache der Vorentwürfe in heutigem, verständlichem Deutsch wiederzugeben. ${ }^{{ }_{5}}$

$\mathrm{Zu}$ fragen bleibt allerdings, ob man noch von einer Obersetzung des lateinischen "Originals" sprechen kann, wenn „in mehr als der Hälfte“ des Textes anderssprachige Fassungen zugrunde gelegt wurden. Letztlich gibt es für dieses Dokument nicht die "Urfassung"; auch der lateinische Text ist - wie die anderen - eine „Übersetzung".

Uber das Ergebnis ihrer Arbeit urteilen die Beteiligten recht positiv: „Auf diese Weise entstand eine deutsche Obersetzung, die von Mitgliedern der päpstlichen Kommission als beispielhaft gewertet wurde."7 Ein nichtbeteiligter Fachmann erwähnt

Friedhelm Geller ssce studierte Theologie, Publizistik und Englisch und ist zur Zeit Studienreferendar für das Lehramt am Gymnasium in Dortmund. 
zwar die "Anmutungen zu Hörfunk und Fernsehen ", gibt aber dem deutschen Text insgesamt eine gute Zensur: „Eine ziemlich einheitliche und fachlich befriedigende Terminologie, die von volkstümlichen Vereinfachungen ebenso weit entfernt ist wie vom Kommunikationssoziologen-Chinesisch. ${ }^{\text {"8 }}$

Gleichwohl schien es lohnend, die deutsche Ubersetzung - in einigen konkreten Beispielen - mit fünf anderen Übertragungen ${ }^{9}$ in Vergleich zu setzen. Dies geschieht punktuell, beschränkt auf Fragen der Fachterminologie und "katholischer Publizistik". Ein solcher Vergleich ist nichts weiter als eine bescheidene Fußnote zu den verschiedenen Beiträgen über die Instruktion. ${ }^{10}$

Praktisch wurde so verfahren, daß - in der Abfolge der Instruktion - unter der jeweiligen Abschnittsnummer des vatikanischen Dokumentes der zu vergleichende Passus zunächst deutsch (D) aufgeführt wird; darunter dann die entsprechende lateinische (L), englische (E), spanische (S), italienische (I) und französische (F) Fassung. Der deutsche Text gibt den Sinnzusammenhang, bei den anderen Versionen werden jeweils nur die notwendigen Begriffe des Vergleichs angeführt. Auf diese Weise kann man leicht die Entsprechungen oder Abweichungen übersehen und die besonderen Abhängigkeiten - etwa des deutschen vom lateinischen Text - feststellen.

Den Zitaten folgt in den meisten Fällen ein kurzer Kommentar; einige Vergleiche deuten sich selbst. Im Kommentar sind $Z$ itate aus den Texten kursiv gedruckt, deren deutsche Übersetzungen stehen in Anführungszeichen.

\section{II.}

Titel

D: Pastoralinstruktion „Communio et Progressio ${ }^{*}$ über die Instrumente der sozialen Kommunikation veröffentlicht im Auftrag des II. Vatikanischen Okumenischen Konzils

L: Instructio Pastoralis ad Decretum Concilii Vaticani II de Instrumentis Communicationis Socialis rite applicandum de mandato eiusdem Concilii exarata.

E: Pastoral Instruction "Communio et Progressio ${ }^{*}$ on the means of Social Communication written by order of the Second Vatican Council.

S: Instrucción Pastoral „Communio et Progressio ${ }^{\infty}$ sobre los medios de Comunicación Social preparada por mandato especial del Concilio Ecuménico Vaticano II.

I: Istruzione Pastorale "Communio et Progressio ${ }^{\alpha}$ sugli strumenti della Comunicazione Sociale pubblicata per disposizione del Concilio Ecumenico Vaticano II.

F: Instruction Pastorale .Communion et Progrès" sur les moyens de Communication Sociale élaborée par mandat spécial du Concile Oecuménique Vatican II.

D und I entsprechen sich genau; im $\mathrm{L}$ wird das Ziel der Instruktion hinzugefügt: "zur rechten Anwendung des Konzilsdekrets“; im E fehlt „ökumenisch“. Der "Name“ der Instruktion ist nur im F übersetzt. Sie wurde im - "besonderen“" (F) - Auftrag des Konzils veröffentlicht (D, I), „entworfen “ (L), "geschrieben * (E), , vorbereitet" (S), „erarbeitet" (F).

1.

D: Gemeinschaft und Fortschritt der menschlichen Gesellschaft

L: communio et progressio convictus humani

E: the unity and advancement of men living in society

S: la comunión y el progreso en la convivencia bumana

I: la comunione e il progresso della società umana

$\mathrm{F}$ : communion et progrès dans la société 
Im D scheint hier eine gute Lösung gefunden; möglich wären auch „Einheit und Entwicklung" wie im E. Dort hätte auch „communion and progress" verwendet werden können. Gesellschaft, società und société sind abstrakter als convictus, das nicht so sehr eine formierte Gesellschaft als vielmehr „geselliges Zusammenleben, geselligen Umgang “ - vor allem beim Gastmahl - bezeichnet. ${ }^{11}$ Auch die englische und spanische Übersetzung sind hier weniger formell.

4.

D: ... diese Instruktion unter Hinzuziehung von Sachverständigen und der zuständigen Stellen auf diözesaner, nationaler und internationaler Ebene . . . in die Tat umzusetzen.

L: consiliorum dioesanorum . . .

$\mathrm{E}$ : diocesan ... councils

S: organismos diocesanos...

I: uffici diocesani ....

F: Conseils diocésains ... .

Der unbestimmte Begriff Stellen (D) wird im L, E und F konkreter wiedergegeben mit dem, was vermutlich gemeint ist: „Räte “ der Diözesen usw. Durch Großschreibung - Conseils - wird im F offensichtlich auf die schon bestehenden Organe kirchlicher Mitverwaltung hingewiesen.

5.

D: Schließlich darf man hoffen, daß diese Instruktion auch bei denen Zustimmung findet, die im Bereich der sozialen Kommunikation in irgendeiner Weise beruflich tätig sind.

L: officio aliquo perfungantur

E: who are professionally involved

S: que se ocupan de alguna manera

I: che exercitano un compito professionale

F: ceux dont la fonction s'exerce

D, E, I beschränken sich hier auf Berufs-Kommunikatoren; die anderen Ubersetzungen sprechen von denen, die „in irgendeiner Weise“ im Bereich der Medien arbeiten.

6.

D: [Die Medien] öffnen Geist und Herz für die gegenwärtige Zeit

L: praesentisque temporis animum aperiunt et mentem

$\mathrm{E}$ : inform about what goes on in the world

S: mostrando el estilo y mentalidad del nuestro tiempo

I: dànno la chiave per comprendere la mentalità del tempo presente

F: apportent une image de la vie du monde d'aujourd'bui

Das weihevolle öffnen Geist und Herz entspricht dem $\mathrm{L}$ und wird in den anderen Ubersetzungen einfacher und unpretentiöser wiedergegeben: „informieren über das, was in der Welt vorgeht" ${ }^{*}(E)$. D, I deuten den doppelsinnigen lateinischen Genitiv final: für die gegenwärtige Zeit, „um . . zu verstehen“, die anderen Versionen interpretieren hier im Sinn der Spiegelungshypothese: die Medien geben ein „Bild“ (F) der Zeit, „zeigen (deren) Stil und Mentalität“ (S).

10.

D: [Christus] begann das Gespräch mit den Menschen

L: commercium haberet cum hominibus

$\mathrm{E}$ : made the first move to make contact 
S: inició la comunicación

I: prese per prima l'iniziativa . . col ristabilire un dialogo

F: prend l'initiative du contact

Commercium, contact, comunicación sind umfassender als Gespräch oder dialogo.

11.

D: [Christus] als Meister der Kommunikation

L: perfectum Communicatorem

E: perfect Communicator

S: perfecto „Comunicador

I: perfetto Comunicatore

F: parfait „Communicateur ${ }^{\alpha}$

Im $S$ und F Distanz zu dieser ungewohnten Bezeichnung durch Anführungszeichen. Die gewählte deutsche Formulierung ist sicher besser als das wörtliche „vollkommener Kommunikator".

14.

D: ergeben sich die Grundsätze [für den etbischen Gebrauch] aus dem Wesen und der Eigengesetzlichkeit eines jeden Mediums

L: qualitatibus cuiusque instrumenti propriis

$\mathrm{E}$ : the innate qualities of the medium in question

S: de las pecularidades

I: dalle caratteristiche proprie

F: des qualités propres

Das D ist hier im Wesen und Eigengesetzlichkeit "philosophischer" als die anderen Ubersetzungen: „Eigenschaften ${ }^{\star}$, „Besonderheiten ${ }^{\star}$.

15.

D: Alle Kommunikatoren (d.h. solche, die sich beruflich der Kommunikationsmittel bedienen) sind verpflichtet, sich Kenntnis und Wissen anzueignen ...

L: illos qui pro munere suo ... utuntur

E: who actively employ

S: los profesionales

I: coloro cioè che per impegno professionale

$\mathrm{F}$ : les divers producteurs

Hier - wie schon in Nr. 5 - eine sehr unterschiedliche Interpretation der Adressaten: beruflich (D), „die ... bei ihrer Arbeit gebrauchen“ (L), „die aktiven Gebrauch machen “ $(E)$, „die Produzenten“ $(F)$.

D: Rezipienten (Leser, Hörer und Zuschauer)

$\mathrm{L}$ : receptores (i.e. illi qui legendo audiendo intuendo utilitates inde bauriunt)

$\mathrm{E}$ : „recipients" are those who, for their own purpose, read, listen to or view the various media

S: "los receptores" (esto es: los que se benefican de lo que leen, oyen o ven)

I: $i$ recettori (coloro cioè che leggendo, ascoltando, guardando, usano questi strumenti)

F: usagers - c'est-à-dire ceux qui lisent, entendent ou regardent

Anführungszeichen im E, $\mathrm{S}$ zeigen die Neuheitsqualität des Begriffs recipient/ receptor. In Unterscheidung $\mathrm{zu}$ den beschreibenden Definitionen von $\mathrm{D}, \mathrm{I}, \mathrm{F}$ sehen L, E, S den Rezipienten positiv motiviert: er rezipiert "zum eigenen Nutzen . 
16.

D: Information, Bildung, Unterbaltung

L: nuntios suos et artes et oblectamenta

E: news, culture and entertainment

S: sus noticias, su arte $y$ sus diversiones

I: notiziari, le trasmissioni culturali e quelle ricreative

F: informations, les émissions artistiques et les divertissements

Das D besitzt mit dem Begriff Bildung eine Vokabel, die das Gemeinte wohl am besten trifft und in den anderen Sprachen kein Äquivalent hat: vgl. culturel culturali, artelartistiques. Das lateinische ars bezeichnet »jede körperliche oder geistige Fertigkeit «12, während arte (S) oder art(istique) (F) lediglich unseren Begriff »Kunst« decken.

17.

D: Nicht allein das Thema oder die vertretene Meinung bestimmen den sittlichen Wert einer Kommunikation, sondern auch der Geist, aus dem heraus sie geschieht.

L: Laus et praestantia moralis .. . non emerget ex solo argumento, neque ex doctrina eius mente tantum concepta, sed etiam ex ratione qua communicatur.

$\mathrm{E}$ : The moral worth and validity of any communication does not lie solely in its theme or intellectual content. The way in which it is presented . . .

S: La estima y valor moral de una comunicación no nace sólo de su contenido ni de su enseñanza teórica, sino también del motivo que la determina.

I: La validità e la moralità di una comunicazione non derivano soltanto dalla bontà dell'argomento né dall'intento dottrinale di chi l'ha concepita. Sono fattori essenziali anche il modo di impostare...

F: Le mérite et la valeur morale d'une information ou d'une émission ne dépendent pas seulement $d u$ sujet traité, ni de la doctrine qui $y$ est implicitement contenue, mais aussi du genre adopté.

Thema und vertretene Meinung werden in den anderen Sprachen recht unterschiedlich wiedergegeben: "Themen und intellektueller/geistiger Inhalt" (E), beabsichtigte (L, S, I) oder faktisch gegebene „Belehrung ${ }^{\star}$ (D, E, F). Ex ratione ist wohl am besten als "Grund/Motiv" wiederzugeben, wie es im S geschieht. Das deutsche Geist ist $\mathrm{zu}$ vage und hochgegriffen. E, I sprechen von der "Art und Weise“ der Darbietung (way, modo), F rekurriert auf „Gattung“.

In allen Übersetzungen, außer der deutschen, ist von einem zweifachen Urteilskriterium die Rede, vom "moralischen Wert“, d.h. der der Kommunikation inhärenten Bedeutung und der Validität (laus, validity, estima, validità, mérite), der von außen herangetragenen Anerkennung oder festgestellten Ubereinstimmung zwischen Aussage und Wirklichkeit.

20.

D: Nachricht und Information

L: rei iudicium seu cognitio

$\mathrm{E}$ : a mass of information

S: rumor o noticia

I: informazioni

F: l'information

Das L schließt hier auch den Kommentar ein, "Urteil über eine Sache“, E, I, F reduzieren den tautologischen Doppelbegriff zu einer Aussage, $S$ hat wörtlich: "Gerücht und Nachricht". 
D: Bildung und Unterweisung auf allen Ebenen [zieben Nutzen aus den Medien]

L: traditio quoque doctrinae cuiusvis gradus

$\mathrm{E}$ : teaching at all levels

S: enseñanza en calquier grado

I: l'istruzione di qualsiasi grado

F: l'enseigment à tous les degrés

Gemeint ist wohl "Unterricht" jeder Art, Bildung trifft nicht; sie ist das Ergebnis dessen, wovon hier die Rede ist und das die anderen Ubersetzungen richtig wiedergeben.

21.

D: in einer pluralistischen Gesellschaft

L: in populis pluralisticis

$\mathrm{E}$ : in a society that is committed to the right of dissent

S: en una sociedad „pluralista ${ }^{\alpha}$

I: dove esiste nella vita sociale una impostazione pluralista

F: chez les peuples qui admettent la liberté et la diversité des opinions

Pluralistische Gesellschaft findet sich nur im D und S. Zwei Ubersetzungen definieren den Begriff: „eine Gesellschaft, die dem Recht auf Meinungsverschiedenheit verpflichtet ist".(E), "wo im gesellschaftlichen Leben eine pluralistische Aufgabe besteht “ (I). Dem lateinischen »Original « — , in pluralistischen VöIkern“ - entspricht hier das F am besten: „bei den Völkern, die Freiheit und Verschiedenheit der Meinungen zulassen “.

D: [Die Instrumente] wenden sich . . an möglichst viele Empfänger

$\mathrm{L}$ : ad quam plurimos utentes

$\mathrm{E}:$ a mass audience

S: al mayor número posible de receptores

I: ad un grosso pubblico indiscriminato

F: multitude d'usagers

Das $\mathrm{E}$ ist präzise und gebraucht den Fachbegriff „Massenpublikum“; die anderen Ubersetzungen suchen eine Entsprechung für das lateinische „möglichst viele Benutzer*.

D: Wie läßt sich vermeiden, daß durch die Konzentration der Medien in den Händen ganz weniger...

L: pauci homines ita instrumentis dominentur

$\mathrm{E}$ : concentration of the power to communicate

S: un uso excesivo

I: un monopolio dominato da pochi

F: la concentration de ces moyens

L:. . . daß „wenige Menschen die Medien so beherrschen . . . ; E: „Konzentration der Kommunikationsmacht“; S: „übermäßiger Gebrauch“; I: „ein von wenigen beherrschtes Monopol ${ }^{\star}$ (eine Tautologie!); F: „Konzentration dieser Mittel ${ }^{\alpha}$.

D: Die Medien verführen bäufig den Menschen dazu, sich in eine Traumwelt zu flüchten.

L: invitent ... ad alio effugiendum per somnium quoddam

$\mathrm{E}$ : invite... to escape into fantasy

S: invitan ... a la evasión

I: invitano ... ad evadere dalla realtà quasi come in un sogno

F: incitent ... à l'évasion et à la rêverie 
Das negative verführen (D), das eine bestimmte Sicht der Medien verrät, ist in allen anderen Ubersetzungen neutral: die Medien „laden ein " (L, E, S, I), "treiben $\mathrm{zu}^{\prime \prime}(\mathrm{F})$.

22.

D: Ansicht, daß die Kommunikationsmittel nur den . . . Zustand der menschlichen Gesellschaft widerspiegeln ...

L: relucere ac referri mores

E: only a reflection of what already exists in society

S: el reflejo de los costumbres de la sociedad humana

I: respecchiare e registrare $i$ costumi già in atto nella società

F: [fehlt]

„Sitten/Gebräuche“ (S, I) decken das lateinische mores nicht ab; Zustand (D) und "was schon in der Gesellschaft vorhanden ist ${ }^{\alpha}$ (E) bleiben vage, weil sie den im I mitgemeinten - Bezug zum Menschen nicht genügend verdeutlichen. Im F fehlt dieser Passus, und Nr. 22 ist insgesamt um die Hälfte kürzer.

$D:$ in diesem Bemühen [den sittlichen Normen wieder Geltung zu verschaffen] fällt den Kommunikationsmitteln eine unverzichtbare Rolle zu.

L: haud mediocres sustinent partes

E: have no small part to play

S: les corresponde no poco a los medios

I: possono offrire un valido aiuto

F: on ne saurait négliger le róle des media

Unverzichtbare Rolle ist in den anderen Versionen weniger dramatisch wiedergegeben: „sie haben keine kleine Aufgabe (L, E, S), „können eine wirksame Hilfe bieten " $(\mathrm{I})$, "man sollte die Rolle der Medien nicht vernachlässigen " $(\mathrm{F})^{13}$.

24.

D: Kommunikationsmittel bilden... ein öffentliches Forum, auf dem das Gespräch der Menschen hin und ber geht.

L: ubi homines ultro citroque habeant sermones

$\mathrm{E}$ : where every man may exchange ideas

S: donde los hombres reciprocamente se hablan

I: dove gli uomini possono interpellarsi e rispondersi

F: où l'on échange des nouvelles, où s'expriment et s'affrontent de multiples opinions

Die deutsche Formulierung ist Henk Prakke verpflichtet ${ }^{14}$ und entspricht dem L, mit dem Unterschied, daß im D - auch S, F - das "Gespräch" als Faktum hingestellt wird, während es in den restlichen Übersetzungen lediglich als Möglichkeit erscheint: Konjunktiv im L, may (E), possono (I). Das F ist hier ausführlich-interpretierend: „Wo man Nachrichten austauscht, wo vielfältige Meinungen ausgesprochen werden und sich gegenüberstehen ${ }^{*}$.

33. [Öberschrift]

D: Das Recht auf Information

L: Ius , informationis ${ }^{\mathrm{T}}$ consequendae atque impertiendae

$\mathrm{E}$ : The right to be informed and to inform

S: El derecho a obtener y comunicar la información

I: Diritto di ricevere e di dare informazioni

F: Droit d'être informé et d'informer

Die nicht-deutschen Ubersetzungen präzisieren: „Recht, Information zu empfangen und zu geben". 
41.

D: die Empfänger

L: receptores

E: recipients

S: el público

I: i recettori

F: ceux qui reçoivent

In Unterscheidung zu Nr. 15 gebraucht D hier Empfänger, dort Rezipienten, das $S$ „Publikum“, dort „receptores“, das $F$ „diejenigen, die empfangen“, dort „die Benutzer“.

42.

D: das Recht auf Unverletzlichkeit des Intimbereichs für die Familie und den einzelnen

$\mathrm{L}$ : ius vitae privatae quod intimum familiarum a simplorum circulum defendit

$\mathrm{E}$ : the right of privacy which protects the private life of families and individuals

S: el derecho a la vida privada, que defiende...

I: diritto alla salvaguardia della vita privata, che defende . . .

F: secrets des individus et des groupes, en particulier des familles

„Privatleben ${ }^{\alpha}$ (L, E, S, I) scheint Intimbereich überlegen. Im F: „Geheimnisst von einzelnen und Gruppen, vor allem der Familien“.

D: das Recht auf Wabrung des Berufsgebeimnisses

L: ius secreti

E: right of secrecy

S: derecho al secreto

I: diritto del segreto

F: secrets professionels

Nur D und F sprechen von Berufsgeheimnis, alle anderen Ubersetzungen vom „Recht auf Geheimnis“ in einem allgemeinen Sinn.

46.

D: Kommunikationsfreibeit

L: libertatem instrumentorum

$\mathrm{E}$ : freedom in the use of the media

S: libertad de los medios

I: libertà nell'uso delle comunicazioni sociali

F: nécessaire indépendance des moyens

D, I: „Freiheit der Kommunikation“, L, E, S: „Freiheit der Medien“, F: „not. wendige Unabhängigkeit der Medien “.

48. [Überschrift]

D: Erziehung, Bildung, Unterhaltung

L: educatio, animi cultura, et otium

$\mathrm{E}$ : education, culture and leisure

S: educación, cultura y ocio

I: educazione, cultura e divertimenti

F: éducation, culture, loisirs

Alle Ubersetzungen, außer der deutschen und italienischen, übertragen den dritten Begriff mit „Freizeit“. Zu Bildung vgl. Nr. 16 und 20.

D: Apparate für Ton-und Bildübertragung, praktische Ton- und Bildkassetten 
L: auditorii ac visifici apparatus, necnon et commodissimae cistellulae ( cassettes $^{\alpha}$ )

$\mathrm{E}$ : audio-visual aids, the new video cassettes

S: medios audiovisuales, las cintas sonoras y visuales (cassettes)

I: attrezature audio-visive... le commodissime forme di registrazione sonora e visiva chiamate ${ }_{\text {cassette }}$ c

F: les moyens audio-visuels, les video-cassettes

Ein Musterbeispiel für die Schwierigkeit, moderne Begriffe ins Lateinische zu übertragen: ১Oft mußten einfache, fachsprachliche Ausdrücke oder Aussagen in komplizierten Umschreibungen wiedergegeben werden ${ }^{15}$ Verwunderlich, daß auch der deutsche und italienische Text diese Schwerfälligkeit nachzuahmen versuchen, die E, S, F mit dem heute üblichen Vokabular richtig und - für jedermann - verständlich übersetzen. Siehe auch Nr. 136.

D: Regeln staatsbürgerlichen Verbaltens [werden vermittelt]

L: regulas complures prosperandae totius communitatis

E: forms of community development

S: normas de la promoción comunitaria

I: indicazioni per lo sviluppo della communitd

$\mathrm{F}$ : enseignement . . . du développement communautaire

D führt den Staat ein, der nirgendwo sonst erscheint, und unterschlägt ${ }_{n}$ die Entwicklung/Förderung der Gemeinschaft".

53.

D: der dauernde Konsum seichter Unterbaltung kann . . a abstumpfen

L: continua . . spectatio operum leviorum

$\mathrm{E}$ : unrelieved diet of productions geared to the lowest cultural level

S: continuada contemplación de obras un tanto superficias

I: la frequenza a spettacoli leggeri

F: le spectacle continuel d'oeuvres de niveau médiocre

Das D ist hier wiederum schärfer und negativer als die anderen Texte: „ein wenig oberflächliche Werke ${ }^{\alpha}(\mathrm{S})$, "leichte Schauspiele“ (I), "Werke von mittelmäßigem Niveau“ (F); E: „abwechslungslose Kost von Produktionen, die auf unterster kultureller Ebene angesiedelt sind ${ }^{\alpha}$.

L, S, I, F sprechen hier nur von „Betrachtung“, das im D mit Konsum wiedergegeben wurde.

D: Wenn die Kommunikatoren selbst um gute Bildung bemübt sind und sich darüber binaus die Fähigkeit erworben haben, auch anderen die Werte der Kultur zu erscbließen,...

L: amplam cognitionem ipsius artis educandi

$\mathrm{E}$ : and buttress their good intentions with a sound knowledge of the science of education

S: que añade . . un amplio conocimiento del arte de educar

I: uniranno una vasta cognizione dell'arte di educare

F: s'ajoute une réelle compétence pédagogique

D übersteigert; es geht um die Forderung, daß der Kommunikator auch ein guter Pädagoge sein müsse. Die Inhalte der pädagogischen Bemühung - Werte der Kultur - erscheinen nur im D.

58.

D: dem Künstler mag das ganze Leben mit allen guten und schlechten Seiten vertraut sein

L: artifici . . observatur ante oculos tota vita bumana

$\mathrm{E}$ : the artist is faced with life in this entirety 
S: el artista contempla la vida bumana entera

I: l'artista ba ben chiaro dinanzi a sé il quadro della vita

F: l'artiste est confronté avec la vie toute entière

Der deutsche Text äußert Zweifel, ob der Künstler die ihm in den anderen Ubersetzungen zugesprochene Fähigkeit besitzt; der Zusatz mit allen guten und schlechten Seiten findet sich nur im D.

75.

D: Nachrichtenredakteure

L: nuntiatores

E: communicators

S: informadores

I: coloro que trasmettono le notizie

$\mathrm{F}$ : les journalistes

76.

D: . . . daß man mit den Medien . . . ein massenbaft großes Publikum erreicht

L: magna et paene interminata bominum frequentia

$\mathrm{E}$ : makes their audience a vast one

S: una cantidad inmensa e indeterminada de personas

I: vasta e quasi sterminata cerchia di uomini

F: vaste public

Schlechtes Deutsch! Ein „großes Publikum ${ }^{\alpha}(F)$ oder „ein möglichst großes Publikum" wäre den Vorlagen, die differenzierter sind, gerecht geworden.

77.

D: [Kommunikatoren, die sich] am wankelmütigen Geschmack der Menge orientieren [leisten einen schlechten Dienst]

L: cupiditate popularis aurae

E: easy popularity

S: ansia de la popularidad

I: popolarità superficiale

F: popularité facile et passagère

Im D eine negative Bestimmung des Publikumsgeschmacks. (Vgl. Nr. 53.) Gemeint ist die Jagd nach "leichter" ${ }^{\text {" }}$ (E, F), „oberflächlicher“ und „unbeständiger Popularität ${ }^{*}(\mathrm{~F})$ von seiten der Kommunikatoren.

79.

D: [in kollegialer Zusammenarbeit können sich die Kommunikatoren] eine Berufsordnung geben

L: disciplinam aliquam et codicem morum

E: codes of ethics

S: normas o códigos

I: un codice morale

F: une déontologie

Die englische Úbersetzung erinnert an die Codes verschiedener Medienbereiche zur Selbstkontrolle. ${ }^{18}$

81.

D: wird Kommunikation zur Einbahnstraße

$\mathrm{L}:$ in unam partem dirigatur locutio 
E: all the efforts will be useless

S: en un sentido único

I: in una sola direzione

F: d̀ sens unique

Im E eine allgemeine Umschreibung: „werden alle Bemühungen nutzlos“.

84.

D: Alle Bürger und Träger staatlicher Gewalt sind dafür verantwortlich, daß die Kommunikationsmittel dem Fortschritt . . . dienen.

L: universi cives et ipsi magistratus

E: both citizens and public authorities

S: todos los cirdadanos y los legisladores

I: tanto $i$ cittadini che le pubbliche autorita

F: tous les citoyens et les législateurs

Sind Träger staatlicher Gewalt (D), „öffentliche Autoritäten “ (E, I) und „Gesetzgeber $^{\text {" }}(\mathrm{S}, \mathrm{F})$ identisch?

85.

D: eine politisch wache Gesellschaft

L: politica ... communitas

$\mathrm{E}$ : the well-being of society

S: la comunidad politica

I: una vera comunita civile

F: la communauté politique

88.

D: allerdings wird es hier und da nötig sein, daß die öffentliche Hand selbst die Errichtung solcher Kontrollgremien in die Wege leitet.

L: ipsos magistratus publicos erigere buius generi consilia munere invigilandi instructa

$\mathrm{E}$ : the state may bave to intervene in order to set up these advisory boards to supervise the media

S: las mismas autoridades creen organismos de control

I: lintervento pubblico per costituire delle commissioni di vigilanza

F: peut-être . . . les pouvoirs publics constituent des organismes de surveillance

Am schärfsten ist hier die englische Fassung: , ... daß der Staat möglicherweise interveniert, um die Medien zu überwachen "..17 Dem entschiedenen deut-

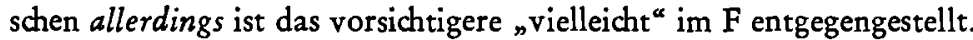

89.

D: [Schutz vor Schäden, die der] seelischen Entwidklung [der Jugend] und ibrem sittlichen Urteil [zugefügt werden können]

L: a magnis aliquando detrimentis diuque mansuris . . . animis atque iudicio honestatis

E: from what can do them permanent psychological or moral harm

S: de los daños tan graves $y$ a veces tan duraderos... en su voluntad $y$ en su criterio moral

I: dai gravi danni di ordine psicologico e morale

F: contre les dommages quelques fois graves et durables... d̀ leur affectivité et à leur sens moral

Die Schadensbereiche sind sehr unterschiedlich umschrieben: seelische Entwicklung/sittliches Urteil (D), „psychologische oder sittliche Schäden * (E, I), „Schaden im Bereich des Willens und des moralischen Urteils“ (S, L), „Affektivität und sittlicher $\operatorname{Sinn}^{*}$ (F). 
103. [Überschrift]

D: Aufgaben der Katholiken auf dem Gebiet der Kommunikationsmittel

L: navitas

E: the commitment

S: acción

I: l'impegno

F: l'action

Das lateinische navitas - 'Rührigkeit, Regsamkeit, Emsigkeit ${ }^{18}$ - wird verschieden interpretiert: Aufgaben (D), „Verpflichtung “ (I), „Engagement ${ }^{*}$ (E), „Handeln ${ }^{\alpha}(\mathrm{S}, \mathrm{F})$; letzteres entspricht der Vorlager am besten.

D: [die Katholiken können belfen, daß bei der] Nachrichtengebung in Presse und Funk religiöse Ereignisse. . . nicht unerwähnt bleiben

L: scriptores et praecones nuntiorum

$\mathrm{E}$ : news editors and newscasters

S: escritores y difusores de noticias

I: quanti curano e trasmettono le notizie

F: ceux qui préparent les nouvelles pour la presse écrite et la presse parlée

Die deutsche Fassung wählt das Abstraktum - bei der Nachrichtengebung wo es doch um ganz konkrete Kommunikatoren geht.

104.

D: Es ist das Recht katholischer Kommunikatoren, von der Kirche geistliche Hilfe zu erhalten, die ihrem ernsten und schweren Beruf entspricht.

L: communicatoribus potestas est . . ut auxilium spirituale . . officium grave et difficile

$\mathrm{E}$ : It is a source of strength for catholic communicators that they receive . . . spiritual belp ... important and difficult role

S: los informadores católicos tienen derecho a recibir . . . la ayuda espiritual . . importancia y dificultad de su misión

I: i comunicatori cattolici hanno diritto di ricevere . . assistenza pastorale . . compito cosi impegnato e difficile

F: les producteurs catholiques ont droit a recevoir . . . l'appui moral . . fonction aussi délicate

Das lateinische potestas est kann verschieden interpretiert werden: 'dürfen, können; Macht, Erlaubnis, Gelegenheit, Möglichkeit habens, wird jedoch nicht im Sinn von "das Recht zu etwas haben " gebraucht, wie es D, E, S, I tun. ${ }^{19}$ Man vergleiche Entsprechungen - Recht auf Information (Nr. 33), auf Privatleben, Berufsgeheimnis (Nr. 42) — wo im L jeweils ius steht. Hier schwächt das „Original" offenkundig seine Vorlagen ab. L wäre richtig zu übersetzen: „Katholische Kommunikatoren können/dürfen . . Hilfe erhalten “.

Der englische Text setzt diese Hilfe als schon gegeben voraus: „Es ist eine Quelle der Stärke für die ... Kommunikatoren, daß sie geistliche Hilfe erhalten ${ }^{*}$. Diese Hilfe ist im I „pastoraler Beistand “, im F „moralische Unterstützung “.

Grave kann sernst' bedeuten, ist aber hier - als Adjektiv zu officium - wohl mit sschwerwiegend, bedeutsam' zu übertragen (vgl. E, S, I).

108.

D: alle formalen und inhaltlichen Probleme der sozialen Kommunikation

$\mathrm{L}$ : cunctae autem res et rationes

$\mathrm{E}:$ the whole question

S: los temas $y$ cuestiones referentes a 
I: la trattazione teorica e prattica

F: tout ce qui touche à la communication

Die deutsche Übersetzung gibt sich — unnötigerweise - „wissenschaftlich“. Warum nicht, wie in den anderen Versionen: „alle Fragen der Kommunikation“?

114. [Überschrift]

D: Der Dienst der Kommunikationsmittel für die Katholiken

L: Communicationis instrumentorum beneficia

E: The Contribution of the communications media

S: Los instrumentos de comunicación al servicio . . .

I: Utilità per $i$ cattolici

F: L'utilité des media pour...

Der deutsche Text ist unklar; gemeint ist, wie die anderen Ubersetzungen zeigen: der Nutzen der Medien für Katholiken.

118.

D: allerdings bringt es die Situation der Massenkommunikation mit sich, daß neue und noch unausgereifte Meinungen von Theologen of am falschen Platz erscheinen.

$\mathrm{L}:$ ex ipsa natura evenit

$\mathrm{E}$ : because of the very nature

S: por la misma naturaleza

I: per la struttura funzionale stessa

F: de fait,...

Situation (D) ist etwas anderes als "Natur “ (L, E, S) oder "funktionale Struktur “ (I). Im F heißt es bündig „tatsächlich“. Der gesamte deutsche Satz ist mißverständlich; gesagt werden sollte: Neue ... Meinungen von Theologen werden häufig vorschnell von den Medien veröffentlicht.

122.

D: das Gespräch der Kirche beschränkt sich nicht auf die Gläubigen

L: fideles

$\mathrm{E}$ : ber own members

S: a sus fieles

I: ai fideli

F: à ses membres

E, F vermeiden „Gläubige “ (vgl. Nr. 140).

136.

D: Kleinschriften und Taschenbücher... Zeichnungen und illustrierte Erzäblungen baben sich als brauchbar erwiesen

L: libelli exiles atque libri sola ex charta confecti . . . imagines lineares atque narratiunculae speciebus collustratae summam peperunt utilitatem

$\mathrm{E}$ : paperbacks, booklets and every sort of leaflet ..., comics“ and illustrated stories are not to be despised

S: sencillas páginas $y$ folletos impresos... grabados $y$ los relatos ilustrados son muy útiles

I: opuscolo $0 .$. un ${ }_{\text {tascabile }}^{\alpha} \ldots$ fumetti ... e $i$ raconti illustrati possono essere utilissime

F: brochures et les livres de podhes... bandes dessinées et de courts récits illustrés présentent une réelle utilité 
Wiederum ein Beispiel für die Ungeeignetheit des Lateinischen, modernes Vokabular klar und adäquat wiederzugeben (s. Nr. 48). L lautet wörtlich übersetzt: „Kleine Büchlein und nur aus Papier hergestellte Bücher [sic] . . Strichzeichnungen und kleine - mit Bildern/Gestalten geschmückte - Erzählungen haben sich als sehr nützlich erwiesen."

D übernimmt Zeichnungen, obwohl "Comics" oder "Comic Strips" gemeint sind (E, I). Mit den illustrierten Erzählungen dürften die, vor allem in Frankreich und Italien verbreiteten, Fotoromane gemeint sein.

$D$ und $E$ übersetzen den „sehr großen Nutzen “ (L, S, F) zurückhaltend-negativ: als brauchbar erwiesen (D), „sind nicht zu verachten ${ }^{\text {" }}(\mathrm{E})$.

137.

D: Tageszeitungen, Magazine oder andere Schriften

L: acta diurna, commentarios, alia scripta

$\mathrm{E}:$ reviews, magazines and periodicals

S: diarios, revistas y todo tipo de publicaciones periódicas

I: quotidiani, riviste, periodici

F: journaux, revues, magazines et périodiques

Acta diurna: die - ab 59 v. Chr. täglich - in Rom veröffentlichten Berichte mit politischen und gesellschaftlichen Nachrichten, die auch in den Provinzen verbreitet wurden und u.a. Tacitus als Quellen dienten. ${ }^{20}$ Die Acta diurna gelten als frühe Vorläufer der Tagespresse.

E läßt "Tageszeitungen" aus, nur im L findet sich commentarios, das mit Magazin (D, E, F) oder „Zeitschrift“ (S, I, F) übertragen wird. "Kommentar/Meinung“ findet sich doch auch in Tageszeitungen und anderen Schriften. Es bleibt unklar, was letztlich damit gemeint ist. „Meinungsblätter“? Ebenso verwundert die Folge revues, magazines, périodiques (F).

138.

D: Nachrichten, Kommentare, Meinungen

L: nuntiorum, commentariorum, opinationum

E: newes, opinions, badkground articles

S: noticias, comentarios, $y$ opiniones

I: informazione, critica, interpretazione

$\mathrm{F}$ : informations, commentaires, opinions

Wodurch unterscheiden sich Kommentare von Meinungen? E versucht einen Ausweg mit "Hintergrund-Berichten “. Man vergleiche die Ubersetzung von commentariorum hier mit der des gleichen Begriffs in Nr. 137.

140.

D: die katholische Presse

L: scripta officio catholicorum exarata

E: catbolic publications

S: publicaciones católicos

I: la stampa d'ispirazione cattolica

F: publications catholiques

Nur D und I sprechen von katholischer Presse, die anderen Ubersetzungen von „Veröffentlichungen “, die nicht notwendigerweise periodisch sein müssen. Erweckt der deutsche Ausdruck die Vorstellung einer fest etablierten Institution, so 
sind die anderen Versionen unbestimmter: „Schriften, die aus der Aufgabe/der Verpflichtung/dem Beruf von Katholiken entstanden sind“; „Presse aus katholischem Geist" (I). ${ }^{19}$

D: Die Gläubigen sind aufgefordert, die katbolische Presse regelmäßig zu verfolgen

L: fideles . . . admonentur et incitantur . . . ut lecticent

E: catholics are encouraged to read ... regularly

S: deben los fieles leer asiduamente

I: fedeli . . . una pressante esortazione a leggere regolarmente

F: la communauté chrétienne es invitée d lire habituellement

E und F sprechen nicht von "Gläubigen“; F: „christliche Gemeinde" (vgl. Nr. 122). Warum wurde im Deutschen nicht auch - wie in den anderen Ubersetzungen - das Wort lesen genommen ${ }^{21}$

142.

D: Der Film hat seinen beredigten Platz und seine Wurzel im Leben der Menschen selbst

L: ludi cinematographici suo prope iure obtinent suasque radices capiunt in ipsa vita bominum

$\mathrm{E}$ : the cinema is part of contemporary life

S: el cine, por derecho propio, ocupa un lugar y está enraizado en la vida de los hombres

I: il cinema si è ormai inserito stabilmente $e$ affonda le radici nella vita contemporanea

F: le cinéma fait partie intégrante de la vie contemporaine

Knapp, verständlich und ausreichend: $E$ und $F$.

144.

D: darf man nicht übersehen, daß viele Filme . . . ausgesprochen religiöse Themen behandelten.

In allen anderen Übersetzungen steht hier das Präsens. Die deutsche Formulierung scheint zu unterstellen, daß es solche Filme heute nicht mehr gebe.

147.

D: . . daß das Filmschaffen als schöner und hoher Beruf anzuseben ist

L: uti pulchram et praestantem professionem

$\mathrm{E}$ : nobility of the vocation of those involved in film production

S: una profesión bonrosa e importante

I: una professione nobile e bella

F: [fehit]

Schöner und hober Beruf vgl. mit ernstem und schweren Beruf in Nr. 104 !

Das seit einigen Jahren bei Cineasten übliche „Filmemachen" hätte wohl in diesem Zusammenhang nicht gepaßt.

148.

D: in Hörfunk und Fernseben werden . . Ausdrucksformen entwickelt, die den Menschen in seinem Innersten bewegen können

L: ipsum bominem immutare valent

E: which can affect man in new ways

S: que pueden cambiar muy profondamente al hombre

I: che possono dare altre dimensioni all'nomo

F: susceptibles de transformer l'homme profondément

Das D hat den sstärksten Ausdrudk. L: „verändern“, E: „in neuer Weise ansprechen“, S, F: „gründlich verändern“, I: „neue Dimensionen eröffnen“. 
161.

D: man wird Theaterschriftsteller anregen ... die religiöse Unruhe und Sehnsucht unserer Zeit ... . darzustellen

L: ut religiosas sollicitudines nostrae aetatis

$\mathrm{E}$ : to set man's religious preoccupation

S: para que saquen . . l la problemática religiosa del bombre de boy

I: la problematica religiosa moderna

$\mathrm{F}$ : les aspirations et les problèmes religieux

Wiederum klingt die deutsche Überarbeitung bombastischer als die anderen Ubersetzungen, die sich mit "Sorgen, Problemen, Fragen ${ }^{\star}$ begnügen. Theaterschriftsteller ist eine nicht sehr glückliche Neuschöpfung.

174.

D: jeder Bischof [soll] einen ständigen „Pressesprecher" baben

L: interpretem seu nuntiatorem stabilem

$\mathrm{E}$ : own official and permanent spokesman or press officer

S: un portavoz o informador fijo

I: un portavoce ufficiale e permanente

$\mathrm{F}$ : son porte-parole ou représentant officiel

D, I haben für zwei Aufgaben nur einen einzigen Begriff; mitgemeint ist hier vermutlich eine Art Public-Relations-Mann, der sich nicht nur auf die Presse beschränkt: „Übersetzer/Interpret “ (L), „Sprecher“ (E, S, I), „Vertreter“ (F).

184.

D: die gesellschaftliche Funktion der Kommunikationsmittel

L: modus . . . quo instrumenta . . . perfunguntur

$\mathrm{E}$ : the functioning . . . within the family of man

$\mathrm{S}$ : cómo los medios cumplen su objetivo

I: modalità ottimali di finalizzazione

F: le fonctionnement

Von gesellschaftlicher Funktion spricht nur die deutsche Obersetzung; E: „Funktionieren in der Menschheitsfamilie",22 L: "Art und Weise, in der sie . . . erfüllen“, S: „Ziel erfüllen“, I: „optimale Gegebenheiten zur Erreichung des Ziels“, F: „Funktionieren ${ }^{\star}$.

187.

D: künftige Entwicklung der sozialen Kommunikation im beginnenden Raum-Zeitalter

L: inita sidera aetas

$\mathrm{E}:$ in a space age

S: la era espacial recién nacida

I: la nuova era spaziale

F: le nouvel âge: celui de la communication sociale

Für die französische Übersetzung bringt nicht das Raum-Zeitalter die entscheidenden Veränderungen, sondern „das der sozialen Kommunikation“.

III.

Der angestellte Vergleich einzelner Stellen läßt - auch mit dem Blick auf das Gesamt aller Ubersetzungen - folgende allgemeine Aussagen zu:

1. Alle sechs Übersetzungen zeigen eine sehr große Ubbereinstimmung und entsprechen einander in Umfang und Inhalt. Abweichungen und Unterschiede beschränken sich auf Einzelheiten und schaffen keine wesentlichen Unterschiede. 
2. Der französische Text ist gegenüber den fünf anderen Ubersetzungen, die in den offiziellen Ausgaben \pm 82 Seiten ausmachen, um etwa 10 Seiten kürzer. Die Kürzungen sind sowohl Auslassungen wie Zusammenfassungen längerer Passagen. (22: Anteil der Medien am „Verfall sittlicher Normen“, 62: Gefahren der Werbung, 72: Ausbildung, 80, 113: Finanzierung.)

3. Der deutsche Text - manchmal auch der italienische und spanische - hält sich am engsten an die lateinische „Vorlage“. Die englische und französische Übersetzung bemüht sich um Befreiung von allzu großer Latinität.

4. Die deutsche Übertragung gebraucht häufig ein anspruchsvolles, hochgegriffenes Vokabular, verkompliziert einfache Sachverhalte $(1,5,15,17,21,22,48,53,84$, $85,89,103,108,118,140,142,147,148,161,184)$, bedient sich einer weihevollen, emotionalen Sprache $(6,14)$ qualifiziert neutrale Tatbestände negativ (21, $53,58,77,144)$ und erliegt dadurch nicht selten der Stereotypie vorgeformter Begriffe. Sie hat allerdings auch Vorteile gegenüber anderen Sprachen $(16,48)$.

Als Beispiel - auch für die Verkürzung im französischen Text - sei Nr. 147 angeführt:

D: Weil diejenigen, welche im Filmwesen beruflich tätig sind, mit vielfachen Problemen und Schwierigkeiten zu kämpfen baben, werden die Katholiken, insbesondere kirchliche Filmorganisationen mit ihnen in einem verständnisvollen Gespräch bleiben. Solche Kontakte werden deutlich zeigen, daß das Filmschaffen als schöner und boher Beruf anzusehen ist, zumal alle überzeugt sind, daß durch den Film den Menschen Wertvollstes geschenkt wird. F: Il est soubaitable que les professionels du cinéma trouvent auprès des organismes catholiques spécialisés une comprébension véritable, préludant à un utile dialogue. [Es ist wünschenswert, daß die beim Film beruflich Tätigen in den - darauf - spezialisierten katholischen Organen wirkliches Verständnis finden, das einen nützlichen Dialog vorbereiten kann.]

5. Es wird deutlich, daß eine Fachterminologie für den publizistischen Bereich noch nicht allgemein etabliert ist $(75,76,79,103,136,137,138,174)$.

6. Das Lateinische kann viele der geforderten Begriffe nur umständlich und ungenau wiedergeben $(20,48)$.

7. Alle Ubersetzungen offenbaren die grundlegende Spannung zwischen traditioneller Kirchensprache und moderner Ausdrucksweise. Das "heutige, verständliche Deutsch" 5 wird dadurch nur zum Teil erreicht.

8. Zusätzliche Probleme erwachsen aus den Schwierigkeiten der Kommunikation verschiedener Sprachen untereinander.

Anmerkungen:

1. Hans Wagner: „Entstehung und Absicht des Pastoralschreibens ${ }^{\star}$, in: Pastoralinstruktion „Communio et Progressio“. Nachkonziliare Dokumentation Bd. 11, Trier 1971, S. $8 \mathrm{ff.}$

2. Ebd. S. 12.

3. Pastoralinstruktion, S. $151-291$.

4. Wagner, a.a.O., S. 14.

5. Anton Kochs: "Zur Geschichte der Pastoralinstruktion ,Communio et Progressio: “, CS $4: 1971,297$.

6. Auch Jesús Iribarren spricht von der lateinischen Ubersetzung als dem „Original“ und dem "Originaltext ${ }^{\alpha}$ in: "Pastoralinstruktion und katholische Berufsorganisationen ${ }^{\text {, }}$ CS $5: 1972,11$. 
7. Wagner, a.a.O., S. 14.

8. Michael Schmolke: „Zehn ideengeschichtliche Beobachtungen zur Pastoralinstruktion ,Communio et Progressio" " CS 4:1971, 301 und 306.

9. Päpstliche Kommission für die Instrumente der sozialen Kommunikation: Pastoralinstruktion ...., Vatikanische Polyglott-Druckerei, o. J. Alle anderen Ausgaben entsprechend, mit den vorn (II, Titel) angeführten Titeln. Der deutsche Text ist mit dem in „Pastoralinstruktion " veröffentlichten identisch (s. Anm. 1. und 3.).

10. Hans Wagner: „Einführung und Kommentar “, in: Pastoralinstruktion, S. 1-150; FranzJosef Eilers u.a. (Hrsg.): Kirche und Publizistik, Dreizehn Kommentare zur Pastoralinstruktion „Communio et Progressio“. Mit dem deutschen Originaltext, Paderborn 1972.

11. Karl Ernst Georges: Ausführliches lateinisch-deutsches Handwörterbuch, Hannover/Leipzig ${ }^{9} 1913$, Bd. 1, Sp. 1671.

12. Georges, a.a.O., Sp. 590.

13. Vgl. Schmolke, a.a.O., 304 .

14. Vgl. ebd., 305.

15. Wagner, a.a.O., S. 14.

16. Iribarren, a.a.O., 7 und 15.

17. Eilers' Behauptung „Der im Deutschen fehlende Satz , die Medien überwachen' findet sich auch im lateinischen Text" (Eilers "Die Pastoralinstruktion im Spiegel der Presse ${ }^{*}$, CS 5:1972, S. 44; in diesem Buch S. 109) ist nicht zutreffend. Der "fehlende Satz ${ }^{\text {“ ist }}$ im deutschen "Kontrollgremium ${ }^{\alpha}$ enthalten.

18. Georges, a.a.O., Bd. 2, Sp. 1111.

19. Ebd., Sp. 1812-1814.

20. The Oxford Classical Dictionary, Oxford 1957, S. 6; Georges, a.a.O., Bd. 1, Sp. 274.

21. Schmolke, a.a.O., 300.

22. Der Begriff wurde verbreitet vor allem durch die berühmte Fotoausstellung "The Family of Man" von Edward Steichen (Museum of Modern Art, New York, 1955).

\section{S U M M A R Y}

The Pastoral Instruction "Communio et Progressio“ was written by several different authors and assembled from texts made in various modern languages. As well as this, the document is the first to be published officially by the Vatican in modern language editions. In the synopsis, the editor makes a comparison between the various editions in Latin, German, English, Spanish, Italian and French. He selects his texts for comparison and usually comments according to the Communications subjects which he feels to be important. The texts are presented according to the official of the Instruction.

\section{R E S U M E N}

La instrucción pastoral „Communio et Progressio" nació a partir de trabajos parciales, escritos en diversos idiomas, y fue en la práctica eclesiástica - por deseo de los obispos de diversos paises - el primer documento que se publicó simultáneamente en varios idiomas. El autor de este artículo analiza - haciendo hincapié en ejemplos concretos - la traducción alemana, comparándola con otras cinco versiones y limitándose concretamente a problemas de terminología especializada y del publicismo católico. Al citar fragmentos de las correspondientes partes numeradas de la instrucción se incluyen para su comparación las versiones alemana, latina, inglesa, española, italiana y francesa de los mismos, de modo que pueden apreciarse fácilmente su correspondencia o discrepancias y puede constatarse la dependencia entre ellos, concretamente del texto alemán respecto del texto latino. 